\title{
IgG in cervicovaginal mucus traps HSV and prevents vaginal Herpes infections
}

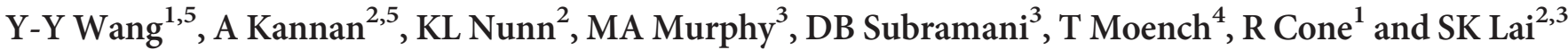

IgG is the predominant immunoglobulin in cervicovaginal mucus (CVM), yet how immunoglobulin $\mathrm{G}(\mathrm{IgG})$ in mucus can protect against infections is not fully understood. IgG diffuses rapidly through cervical mucus, slowed only slightly by transient adhesive interactions with mucins. We hypothesize that this almost unhindered diffusion allows IgG to accumulate rapidly on pathogen surfaces, and the resulting IgG array forms multiple weak adhesive crosslinks to mucus gel that effectively trap (immobilize) pathogens, preventing them from initiating infections. Here, we report that herpes simplex virus serotype 1 (HSV-1) readily penetrated fresh, $\mathrm{pH}$-neutralized ex vivo samples of CVM with low or no detectable levels of anti-HSV-1 IgG but was trapped in samples with even modest levels of anti-HSV-1 IgG. In samples with little or no endogenous anti-HSV-1 IgG, addition of exogenous anti-HSV-1 lgG, affinity-purified from intravenous immunoglobulin, trapped virions at concentrations below those needed for neutralization and with similar potency as endogenous IgG. Deglycosylating purified anti-HSV-1 IgG, or removing its Fc component, markedly reduced trapping potency. Finally, a non-neutralizing IgG against HSV-gG significantly protected mice against vaginal infection, and removing vaginal mucus by gentle lavage abolished protection. These observations suggest that IgG-Fc has a glycandependent "muco-trapping" effector function that may provide exceptionally potent protection at mucosal surfaces.

\section{INTRODUCTION}

Large quantities of immunoglobulin $\mathrm{G}(\mathrm{IgG})$ are transported into female genital tract mucus secretions by the major histocompatibility complex class I-related neonatal Fc receptor, ${ }^{1}$ resulting in at least 10 -fold more IgG than $\operatorname{IgA} .{ }^{2}$ However, despite this predominance of IgG, the precise mechanism(s) by which secreted IgG can prevent vaginal infections are not well understood. Few studies have explored the potential protective role of IgG within the mucus secretions overlaying the epithelial tissue, which sexually transmitted viruses invariably encounter and must penetrate in order to reach target cells. Well-known antibody $(\mathrm{Ab})$ effector functions in blood and lymph (e.g., complement activation, opsonization, and Ab-dependent cellular cytotoxicity (ADCC)) are absent or limited in healthy female genital secretions, which typically have little complement activity and few, if any, active leukocytes. ${ }^{3-5}$ These classical mechanisms of systemic immune protection also do not adequately account for the moderate but significant protection observed in the landmark Thai RV144 HIV vaccine trial. ${ }^{6,7}$ The vaccination regimen modestly reduced the risk of $\mathrm{HIV}$ acquisition despite inducing primarily non-neutralizing $\mathrm{Ab}$ and otherwise offering little to no protection against systemic progression of infections once acquired, suggesting that protection likely occurred before initiation of infection. A better understanding of potential additional mechanisms of vaginal mucosal immunity will also likely be critical for developing effective vaccines against other sexually transmitted infections, including herpes simplex virus (HSV), which has been shown to evade complement and other classical Ab-mediated protective mechanisms. ${ }^{8-10}$

Here, we seek to explore the hypothesis that secreted IgG may have evolved to work in tandem with mucus to trap and thereby exclude individual pathogens. ${ }^{3}$ Viruses must penetrate cervicovaginal mucus (CVM) to reach and infect their target cells in the vaginal epithelium; indeed, we have shown that HIV and human papillomavirus (HPV) are both capable of rapidly

\footnotetext{
${ }^{1}$ Department of Biophysics, Johns Hopkins University, Baltimore, Maryland, USA. ${ }^{2}$ UNC/NCSU Joint Department of Biomedical Engineering, University of North CarolinaChapel Hill, Chapel Hill, North Carolina, USA. ${ }^{3}$ Division of Molecular Pharmaceutics, Eshelman School of Pharmacy, University of North Carolina—Chapel Hill, Chapel Hill, North Carolina, USA and ${ }^{4}$ ReProtect, Baltimore, Maryland, USA. Correspondence: SK Lai (lai@unc.edu)

${ }^{5}$ These authors contributed equally to this work.
} 
diffusing through human genital mucus secretions. ${ }^{11,12}$ We also previously found that the diffusion of IgG $(11 \mathrm{~nm})$ was slowed slightly in human cervical mucus compared with that in saline buffer, while much larger virus-like particles, including the capsids of Norovirus $(38 \mathrm{~nm})$ and HPV $(55 \mathrm{~nm})$, were not slowed by this mucus. ${ }^{12}$ Thus, the slight retardation of the much smaller IgG molecules must be due to very transient $(<1 \mathrm{~s})$, low-affinity bonds with the mucin mesh. ${ }^{12}$ These observations prompt our hypothesis that, by making only transient lowaffinity bonds with mucins, IgG is able to diffuse rapidly through mucus and accumulate on a pathogen surface. The array of $\mathrm{Ab}$ bound to the pathogen surface can in turn effectively trap the pathogen in mucus gel by ensuring at least some low-affinity bonds to the mucin mesh are present at any given time. Virions trapped in CVM cannot reach their target cells and will instead be shed with post-coital discharge and/or inactivated by spontaneous thermal degradation or other protective factors in mucus, such as defensins. ${ }^{13,14}$

\section{RESULTS}

\section{Reduced HSV-1 mobility correlates with increasing endogenous anti-HSV-1 IgG in human CVM}

We chose to explore this trapping-in-mucus hypothesis using HSV-1 $(d \sim 180 \mathrm{~nm})$, a highly prevalent sexually transmitted virus. We collected fresh, undiluted CVM obtained predominantly from donors with normal lactobacillus-dominated vaginal microbiota, as confirmed by Nugent scoring (see Supplementary Table S1 online). HSV-1 virions expressing a VP22-GFP (green fluorescent protein) tegument protein construct, packaged at high copy numbers while maintaining native viral envelope integrity, were mixed into CVM $\mathrm{pH}$ neutralized to mimic neutralization by alkaline seminal fluid. We then performed time-lapse microscopy of virion motions in real time with high spatiotemporal resolution and quantified virion mobility using multiple particle tracking over a long timescale. We observed substantial differences in HSV-1 mobility in CVM samples from different donors (Figure 1a and Supplementary Videos S1 and S2): in 7 of the 12 CVM samples, most virions diffused distances spanning several microns over the course of $20 \mathrm{~s}$, whereas in the remaining 5 CVM samples the majority of virions were essentially trapped, moving less than their diameter $(<200 \mathrm{~nm})$ in $20 \mathrm{~s}$.

As IgG is the predominant immunoglobulin in human $\mathrm{CVM},{ }^{2}$ we examined whether virion mobility correlated with endogenous virus-specific IgG in all 12 of the CVM samples measured using a whole-virus enzyme-linked immunosorbent assay (ELISA; see Supplementary Table S2). In good agreement with our hypothesis, HSV-1 diffused rapidly through all CVM samples that had little or no detectable endogenous anti-HSV-1 IgG $\left(<0.2 \mu \mathrm{g} \mathrm{ml}^{-1}\right.$; detection limit $0.017 \mu \mathrm{g} \mathrm{ml}^{-1}$ ) at rates only several fold lower than their expected rates in water (Figure 1, Supplementary Figure S1, and Supplementary Table S3). In contrast, in samples with elevated levels of endogenous anti-HSV-1 $\mathrm{IgG}\left(\geq 0.6 \mu \mathrm{g} \mathrm{ml}^{-1}\right)$, most HSV-1 virions were effectively trapped. HSV-1 that was trapped in place over the first 20 -s observation remained
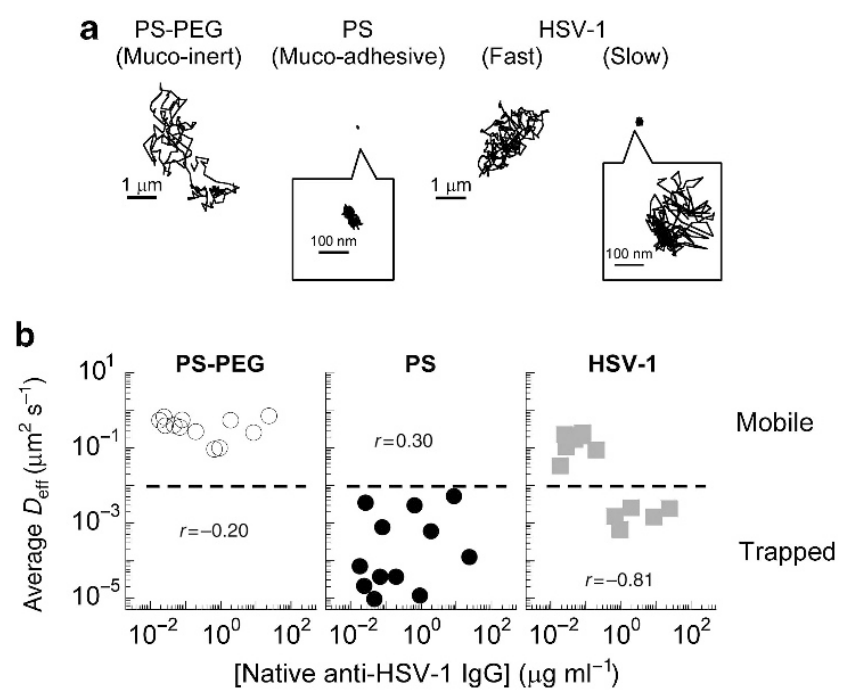

Figure 1 Herpes simplex virus serotype 1 (HSV-1) is immobilized in cervicovaginal mucus (CVM) samples with elevated endogenous antiHSV-1 immunoglobulin G (IgG) but readily mobile in samples with low endogenous anti-HSV-1 IgG. Fluorescent HSV-1 or control particles were added to CVM, and their motions were analyzed by multiple particle tracking methods. (a) Representative $20 \mathrm{~s}$ traces of HSV $-1(d \sim 180 \mathrm{~nm})$ and control particles $(d \sim 200 \mathrm{~nm})$ with effective diffusivity $\left(D_{\text {eff }}\right)$ at a timescale $\tau$ of $1 \mathrm{~s}$ within one s.e.m. of the mean. Control particles include muco-inert (polyethylene glycol (PEG)-coated polystyrene (PS); PS-PEG) and muco-adhesive (uncoated; PS) beads, which are freely diffusive and trapped in human CVM, respectively, as previously shown. ${ }^{15}$ (b) Geometric average $D_{\text {eff }}(\tau=1 \mathrm{~s})$ for PS-PEG, PS, and HSV-1 in individual CVM samples from unique donors $(n=12$, each experiment performed independently) as a function of endogenous anti-HSV-1 IgG. Dashed lines represent the $D_{\text {eff }}$ cutoff below which particles are permanently trapped (moving less than their diameter within $1 \mathrm{~s}$ ). Pearson's correlation coefficients $(r)$ are indicated.

trapped in the same locations for at least $15 \mathrm{~min}$ (see Supplementary Video S3 and Supplementary Figure S2). In the same CVM samples, control latex nanoparticles comparable in size to HSV-1 and engineered with mucoinert coatings (polyethylene glycol-coated polystyrene beads (PS-PEG); $d \sim 200 \mathrm{~nm}$ ) exhibited rapid diffusion (Figure 1, Supplementary Video S4, and Supplementary Figure S1), in good agreement with our previous observations of the large pores present in human CVM (average $d \sim 340 \mathrm{~nm}$ ). ${ }^{15,16}$ Thus, the mucus mesh spacing was large enough for IgG-coated HSV1 (at most $15-20 \mathrm{~nm}$ larger diameter even at saturation) to diffuse relatively unimpeded in the absence of adhesive interactions with mucin gel. Muco-adhesive latex nanoparticles of the same size (PS; $d \sim 200 \mathrm{~nm}$ ) were markedly slowed or immobilized in the same CVM secretions (Figure 1, Supplementary Video S5, and Supplementary Figure S1). Importantly, observations with PS-PEG and PS control particles confirmed that the general barrier properties of all samples, including those with low levels of endogenous antiHSV-1 IgG, remained intact. After removal of 90-95\% of total IgG from these samples by dialysis at constant sample volume, HSV-1 became readily mobile (see Supplementary Video S6 and Supplementary Figure S3), whereas PS beads 
remained immobilized (see Supplementary Video S7 and Supplementary Figure S3). HSV-1 mobility correlated only with endogenous HSV-1-specific IgG and did not correlate with total IgG, IgA, or IgM content (see Supplementary Figure S4).

A well-recognized mechanism of mucosal immune defense is "immune exclusion" in which microorganisms in the gut are agglutinated by secreted polyvalent IgA and IgM into clusters too large to diffuse through mucus. ${ }^{17,18}$ However, we observed little to no agglutinated HSV-1 in our experiments (see Supplementary Video S2, for example), consistent with previous findings that $\operatorname{IgG}$ is a relatively poor agglutinator. ${ }^{19}$ Together, these observations suggest that individual HSV-1 virions in samples with elevated endogenous levels of antiHSV-1 IgG are slowed or trapped by multiple low-affinity bonds with CVM rather than by physical (steric) obstruction.

\section{HSV-1 is trapped by sub-neutralizing levels of exogenously added anti-HSV-1 IgG}

To confirm that trapping of HSV-1 in CVM was mediated specifically by IgG bound to virions and not by any other component in mucus that might be associated with elevated endogenous anti-HSV-1 IgG, we affinity-purified HSV-1specific IgG from human intravenous immunoglobulin (starting with a pure clinical IgG preparation) and mixed the purified IgG into CVM samples that had low endogenous anti-HSV-1 IgG. We found that addition of $1 \mu \mathrm{g} \mathrm{ml}^{-1}$ anti-HSV-1 IgG trapped HSV-1 with a potency comparable to that of endogenous anti-HSV-1 IgG (Figure 2, Supplementary Video S8, and Supplementary Figure S5; $P<0.05$ compared with native specimen without addition of anti-HSV-1 IgG). We further tested lower anti-HSV-1 IgG doses (see Supplementary Videos S9-11) and observed potent trapping of virions when $\sim 333 \mathrm{ng} \mathrm{ml}^{-1}$ anti-HSV-1 IgG was added $(P<0.05)$ and partial trapping when 100 and $33 \mathrm{ng} \mathrm{ml}^{-1}$ antiHSV-1 IgG were added (both $P<0.05$ ). As controls, muco-inert PS-PEG remained freely diffusive and muco-adhesive PS markedly slowed or immobilized in CVM samples treated with the highest anti-HSV-1 IgG doses (see Supplementary Videos S12 and S13, respectively, and Supplementary Figure S6), confirming that the IgG did not cause HSV-1 trapping by altering mucus viscoelasticity or mesh spacing. Affinitypurified anti-HSV-1 IgG exhibited little neutralizing activity at 1 and $\sim 333 \mathrm{ng} \mathrm{ml}^{-1}$ (Figure 2b), based on reduction of plaque formation in Vero cells, suggesting that multiple lowaffinity bonds between IgG and CVM can trap virions at IgG levels lower than those needed to neutralize. HSV-1 was also trapped by a humanized monoclonal anti-gD IgG in CVM (see Supplementary Video S14) but not by control, non-specific IgG (see Supplementary Video S15 and Supplementary Figure S7), underscoring the specificity of trapping via particular $\mathrm{Ab}$-virus pairs, rather than a non-specific interaction or alteration of general mucus barrier properties. In good agreement with previous studies, ${ }^{12,20}$ both polyclonal antiHSV-1 IgG and monoclonal anti-gD IgG were only slightly slowed in CVM compared with saline (see Supplementary
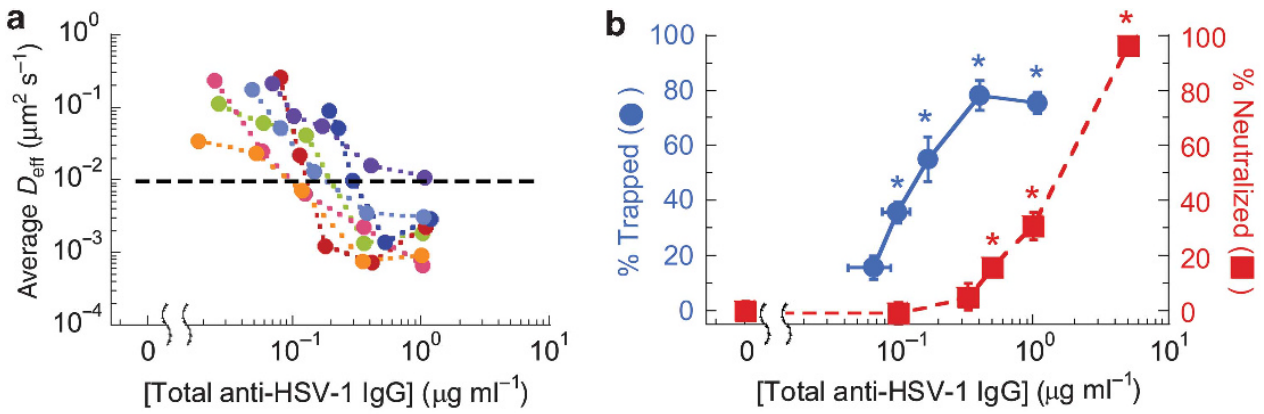

C

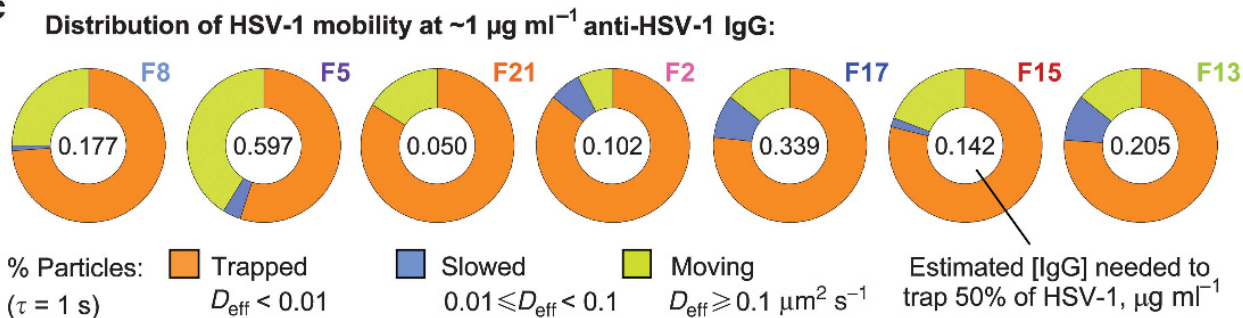

Figure 2 Anti-herpes simplex virus serotype 1 (anti-HSV-1) polyclonal human immunoglobulin G (IgG) added to cervicovaginal mucus (CVM) samples with low endogenous anti-HSV-1 IgG potently traps HSV-1. HSV-1 mobility was quantified in aliquots of the same CVM samples with different amounts of anti-HSV-1 IgG added. (a) Comparison of effective diffusivity $\left(D_{\text {eff }} ; \tau=1 \mathrm{~s}\right.$ ) for HSV-1 in CVM samples ( $n=7$, each experiment performed independently) with different amounts of total anti-HSV-1 IgG (sum of endogenous and added IgG). Different colored circles represent distinct samples. (b) In vitro neutralization vs. trapping potency of anti-HSV-1 lgG. Neutralization was assayed based on reduction of HSV plaque formation in Vero cells; trapping was defined as $D_{\text {eff }}(\tau=1 \mathrm{~s})<0.01 \mu \mathrm{m}^{2} \mathrm{~s}^{-1}$. See Methods section for additional details. Total IgG was averaged across samples for each treatment group. Error bars represent s.e.m. Asterisk $\left(^{*}\right)$ indicates statistically significant difference compared with respective controls $(P<0.05)$. (c) Distribution of particle speeds in samples treated with $1 \mu \mathrm{g} \mathrm{ml}^{-1} \mathrm{lgG}$ (annulus chart), and estimated concentration of total lgG ( $\mu \mathrm{g} \mathrm{ml}^{-1}$ ) needed for $50 \%$ trapping (number in center). Donor ID is indicated for each sample, with colors matching those in panel $\mathbf{a}$. 
Figure S8), suggesting that both Abs form only transient, low-affinity bonds with CVM as individual molecules, yet facilitate effective trapping of virions once they accumulate on the viral surface by forming low-affinity but polyvalent IgG-mucin bonds.

\section{IgG-mucus interaction is dependent on Fc-glycans}

We next sought to determine the biochemical basis of the lowaffinity bonds between IgG and CVM. The Fc domain of all IgGs harbors a conserved N-glycosylation site at Asn297, and many IgG effector functions are Fc- and Asn297 glycandependent. ${ }^{21}$ Thus, we prepared $\mathrm{F}(\mathrm{ab})_{2}$ fragments (Figure 3a and Supplementary Figure S9) and deglycosylated IgG (Figure 3b) from the same affinity-purified anti-HSV-1 IgG to minimize any changes in HSV-1-binding avidity (confirmed by ELISA) and measured the mobility of HSV-1 pre-mixed with these modified analogs before addition to CVM (pre-mixed to minimize interference by endogenous HSV-1-specific IgG). We found both $\mathrm{F}\left(\mathrm{ab}^{\prime}\right)_{2}$ and deglycosylated IgG exhibited substantially reduced trapping potency compared with intact $\operatorname{IgG}$ (Figure 3c; $P<0.05$ ), suggesting that the low-affinity bonds IgG forms with mucins are not only Fc-dependent but also are influenced by Fc glycosylation.

\section{Non-neutralizing monoclonal IgG blocks vaginal HSV-2 infection in mice via IgG-mucus interactions}

To determine whether trapping viruses in mucus can protect against infection in vivo, we evaluated the ability of a nonneutralizing monoclonal $\mathrm{IgG}_{1}$ to reduce $\mathrm{HSV}-2$ transmission in the $\mathrm{pH}$ neutral ${ }^{22}$ mouse vagina. This monoclonal $\operatorname{IgG}_{1}$ bound to the relatively sparse gG surface glycoprotein and exhibited no neutralization activity across all concentrations tested in vitro (Figure 4a); mouse $\operatorname{IgG}_{1}$ also possesses little to no complement ${ }^{23-25}$ and $\mathrm{ADCC}^{26,27}$ activity. We challenged mice
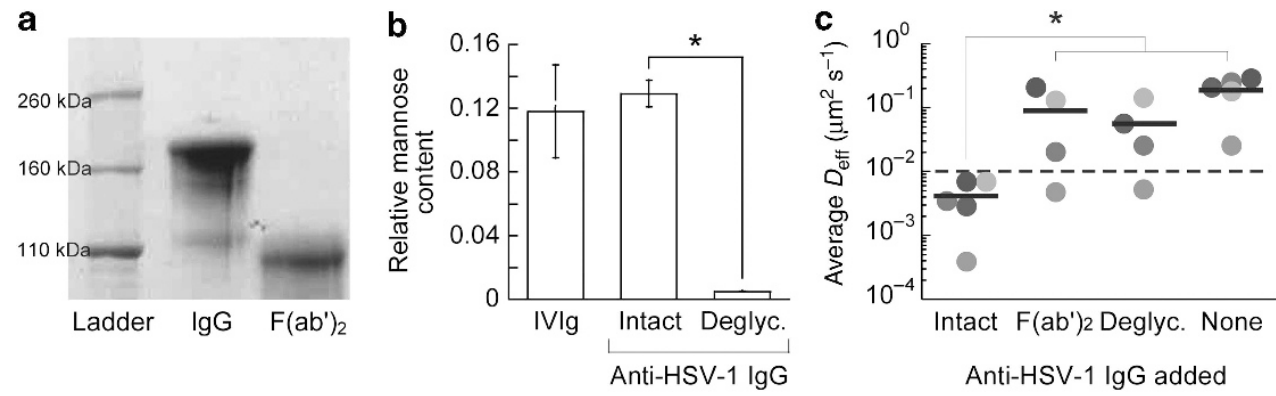

Figure 3 Immunoglobulin G (IgG)-mucin affinity is Fc-and glycosylation-dependent. (a) Preparation of anti-herpes simplex virus serotype 1 (anti-HSV-1) $\left.\mathrm{F}(\mathrm{ab})_{2}\right)_{2}$ confirmed by SDS-PAGE (sodium dodecyl sulfate-polyacrylamide gel electrophoresis); full-length gel is presented in Supplementary Figure S9. (b) Preparation of deglycosylated anti-HSV-1 IgG confirmed by lectin-binding assay (absorbance of IgG-bound ConA normalized to amount of IgG). Error bars represent s.e.m. (c) Mobility ( $D_{\text {eff }} ; \tau=1 \mathrm{~s}$ ) of HSV-1 in cervicovaginal mucus (CVM) with low endogenous anti-HSV-1 IgG incubated with various HSV-1-specific Ab: $1 \mu \mathrm{g} \mathrm{ml}^{-1}$ affinity-purified native IgG ("Intact"), $667 \mathrm{ng} \mathrm{ml}^{-1} \mathrm{~F}\left(\mathrm{ab}\right.$ ') ${ }_{2}$, and $1 \mu \mathrm{g} \mathrm{ml} \mathrm{m}^{-1}$ deglycosylated IgG compared with HSV-1 in native CVM ("None"). Distinct samples ( $n=4-5$, each experiment performed independently) are indicated with different color circles; averages are indicated by solid lines. Dashed line represents the $D_{\text {eff }}$ cutoff below which particles are permanently trapped (moving less than their diameter within $1 \mathrm{~s}$ ). Asterisk $\left(^{*}\right)$ indicates statistically significant difference $(P<0.05)$. A full color version of this figure is available at the Mucosal Immunology Journal online.
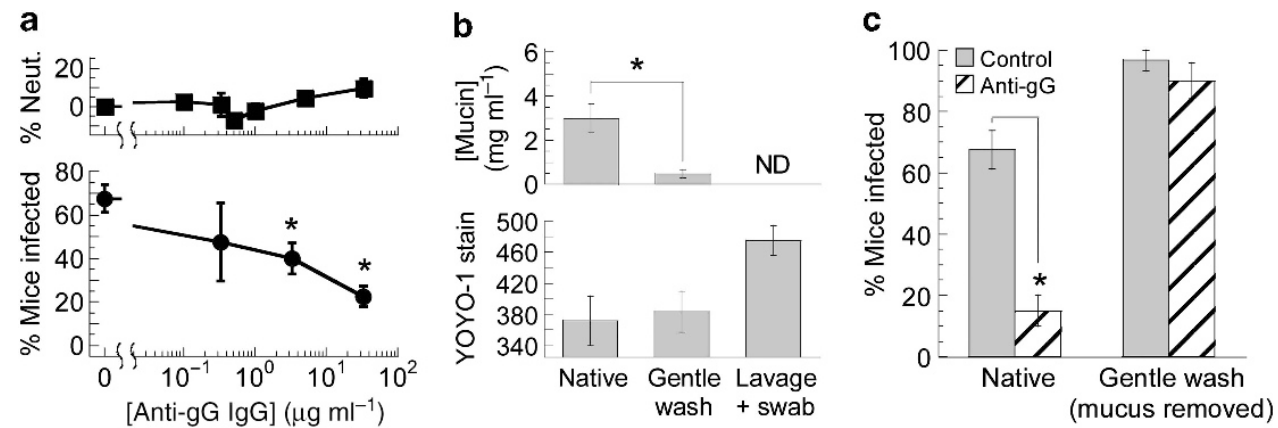

Figure 4 A non-neutralizing monoclonal immunoglobulin $\mathrm{G}(\mathrm{lgG})$ against the $\mathrm{gG}$ epitope protects against vaginal herpes simplex virus serotype 2 (HSV2) infection in mice via IgG-mucus interactions. (a) In vivo protection vs. in vitro neutralization (\% Neut.) of HSV-2. Neutralization was assayed based on reduction of HSV plaque formation in Vero cells. Depo-Provera-treated mice were inoculated with HSV-2 mixed with control or anti-gG IgG. Infection was assayed 3 days post inoculation by detection of virus in vaginal lavages using the ELVIS HSV Test System. (b) Mucin concentration in vaginal fluid collected from the native or gently washed mouse vagina, and YOYO-1 staining for vaginal epithelial cell damage in gently washed or conventionally lavaged and swabbed (cotton tip) mice. ND, no data. (c) In vivo protection by $33 \mu \mathrm{g} \mathrm{ml}^{-1}$ anti-gG IgG is lost when mouse cervicovaginal mucus is removed by gentle washing using a syringe pump. Data represent at least three independent experiments, each with $n=10$ mice per group (in vivo; total $n=40$ per group for data in panel $\mathbf{a}, n \geq 30$ for $\mathbf{c}$ ) or performed in triplicate (in vitro). Error bars represent s.e.m. Asterisk (*) indicates statistically significant difference compared with control $(P<0.05)$. 
vaginally with $2 \mathrm{ID}_{50}$ (50\% infectious dose) HSV-2 with and without anti-gG IgG $\mathrm{Ig}_{1}$ and assayed HSV infection by detection of virus shedding in vaginal lavages 3 days post inoculation, a more sensitive assay of infection than visual observation of lesions, viral isolation from sacral ganglia, or death. ${ }^{28}$ Anti-gG $\mathrm{IgG}_{1}$, at a concentration of $\geqslant 3.3 \mu \mathrm{g} \mathrm{ml}^{-1}$, significantly protected against infection and reduced the average viral load compared with either medium alone or control, non-specific IgG (Figure 4a and Supplementary Figure S10, $P<0.05$ ). Interestingly, anti-gG $\operatorname{IgG}_{1}$ appeared to only reduce the rate of successful vaginal HSV transmission; in mice that became infected, the extent of vaginal infection was comparable to that in mice receiving control IgG, suggesting that the anti-gG IgG dosed did not elicit effector functions that reduced the extent of virus spread in infected mice compared with control IgG (see Supplementary Figure S10).

We also evaluated protection in mice that received a gentle vaginal wash to remove mucus without detectable trauma to the epithelium (Figure $\mathbf{4 b}$ and Supplementary Figure S11). The removal of CVM increased susceptibility to HSV-2 in control mice from $\sim 70 \%$ to $\sim 100 \%$ but not the degree of HSV shedding in mice that became infected (see Supplementary Figure S10). This moderate ( $\sim 30 \%)$ increase in susceptibility is likely attributed to loss of innate protection by CVM itself: a CVM layer prevents immediate direct contact between viruses and the epithelium and contains factors, such as defensins, that may further contribute to overall reduction of infectious HSV flux to the epithelium. More importantly, removal of CVM completely abolished the $\sim 50 \%$ extra protection (from $\sim 70 \%$ to $\sim 20 \%$ infection) afforded by anti-gG $\operatorname{IgG}_{1}$ in naïve mice, which cannot be attributed to innate immunity (Figure 4c). Consistent with the hypothesis that trapping in mucus may facilitate protection, these results together suggest that much of the observed synergistic enhancement in protection by anti-gG $\mathrm{IgG}_{1}$ when CVM is present most likely occurred before HSV reached target cells, rather than by immune mechanisms that can facilitate protection at the cellular level (e.g., complement or ADCC). Our observations are also consistent with the poor complement and ADCC activity of mouse $\operatorname{IgG}_{1}$, as well as numerous previous studies that have shown that HSV can evade complement and other classical immune-protective mechanisms. ${ }^{8-10,29,30}$ As even a non-neutralizing monoclonal IgG against a relatively sparse surface antigen can afford substantial protection, monoclonals against more abundant surface antigens, such as $\mathrm{gD}$ and $\mathrm{gB}$, or those optimized to maximize interactions with mucus are likely to provide even more potent protection at mucosal surfaces in vivo.

\section{DISCUSSION}

The first evidence of Ab-mucin affinity can be traced back to $>30$ years when Kremer and Jager noted that infertility in humans is often caused by anti-sperm Abs. ${ }^{31,32}$ In cervical mucus samples with high levels of anti-sperm Ab, they found that both individual and agglutinated sperm make no forward progress and shake in place for hours until they die, despite vigorous flagellar motility. More recently, Phalipon et al. ${ }^{33}$ suggested that secretory IgA can aggregate pathogenic Shigella flexneri in mouse nasal mucus secretions via the secretory component, anchoring the bacteria to the mucus gel and thereby "excluding" them from infectious entry. In both of the above instances, the authors assumed that the Abs were attached firmly to the mucins. However, fluorescence recovery after photobleaching (FRAP) experiments by Olmsted et al., ${ }^{12}$ and those here, clearly demonstrate that IgG and other $\mathrm{Ab}$ diffuse rapidly in human genital mucus, slowed only slightly and transiently compared with their diffusion in water, and with no non-recoverable fraction indicative of permanently immobilized Ab. Using a complementary technique called fluorescence imaging of profiles, Saltzman et al. ${ }^{20}$ also showed similar rapid Ab diffusion in human cervical mucus. Such rapid diffusion of individual $\mathrm{Ab}$ molecules in mucus can only be explained by weak and short-lived adhesive interactions between $\mathrm{Ab}$ and the mucin mesh. Thus, trapping of individual sperm or pathogens by specific $\mathrm{Ab}$ in mucus gel most likely reflects multiple weak interactions between mucins and an array of bound $\mathrm{Ab}$. These results contrast sharply with the recent work by Fahrbach et al., ${ }^{34}$ which suggested that endogenous IgG binds tightly to mucins. This discrepancy is likely due to experimental artifacts from the use of fluorescently tagged $\mathrm{Ab}$ to label endogenous $\mathrm{Ab}$ in the latter work, which would create large immune complexes that exhibit far slower diffusivity than individual $\mathrm{Ab}$ molecules, consequently misinterpreted as high affinity binding of individual $\mathrm{Ab}$ molecules to mucins.

The mucin-like $\mathrm{Fc} \gamma$-binding protein $(\mathrm{F} c \gamma \mathrm{BP})$ has also been proposed to serve an immunological role in mucus through its ability to bind strongly to IgG Fc. ${ }^{35}$ Nevertheless, more recent evidence indicates the primary function of $\mathrm{F} c \gamma \mathrm{BP}$ is instead to stabilize gastrointestinal mucus gel by covalently cross-linking Muc2 mucins. ${ }^{36,37}$ An IgG Fc-FcyBP-mucin crosslinking mechanism that relies on strong binding of IgG by Fc $\gamma B P$ also directly contradicts numerous previous efforts that have failed to detect any significant binding of individual $\mathrm{Ab}$ to mucins. ${ }^{3,12,20,38,39}$ Finally, Fc $\gamma$ BP binds broadly to all IgG, and the interaction is thus subject to competitive inhibition. ${ }^{40}$ In our experiments where exogeneous HSV-1-specific IgG was added to $\mathrm{CVM}$, total levels of IgG already present in the samples were 100- to 1,000-fold higher than the HSV-1-specific IgG doses added. Thus, for Fc $\gamma B P$ to be responsible for the observed trapping phenomena, $\mathrm{F} \gamma \gamma \mathrm{BP}$ must have been present in greater molar quantities than native IgG, which is unlikely given that the protein has not been routinely identified in proteomic screens of human genital tract fluid (see Andersch-Bjorkman et al., ${ }^{41}$ who identified Fc $\gamma B P$, vs. others, ${ }^{42-44}$ who did not). In contrast to previous studies, by examining the effect of IgG on virions in mucus gel rather than probing directly for interactions between individual IgG molecules and mucins, we were able to document not only the potent trapping of individual virions by multiple surface-bound IgG (Figure 5) but also that the IgG-mucin interactions are Fc- and glycan-dependent.

We have previously shown that HIV-1 and HSV-1 can be effectively immobilized in acidic, lactic acid-rich CVM (at least 
a Mucus (no anti-HSV IgG)

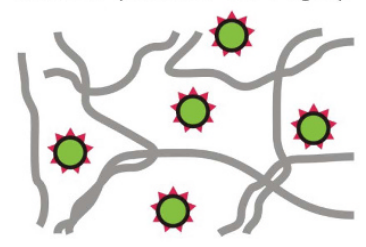

HSV b

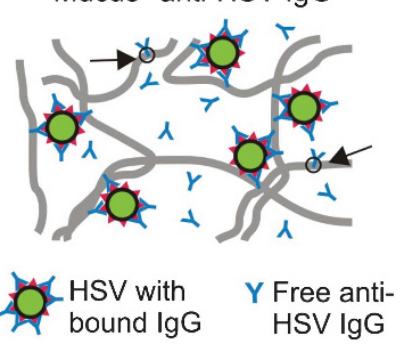

Figure 5 Proposed mechanism of antibody (Ab)-mediated trapping of viruses in mucus. Schematic showing (a) herpes simplex virus (HSV) readily penetrating native cervicovaginal mucus (CVM) with little-to-no endogenous anti-HSV immunoglobulin G (IgG), and (b) anti-HSV IgG trapping HSV in CVM by multiple transient, low affinity bonds with mucins. By forming only short-lived, low-affinity bonds with mucus, free Ab, such as IgG, are able to diffuse rapidly through mucus and bind to viruses. As IgG molecules accumulate on the virus surface, they form multiple low-affinity bonds between the virus and mucus gel. A sufficient number of these transient low-affinity bonds ensure viruses are effectively trapped in mucus at any given time, thereby reducing the flux of infectious virions that can reach target cells. In a similar fashion, IgM, with its 10 relatively lowaffinity Fab components, is well known to make essentially permanent polyvalent "high avidity" bonds. Arrows indicate the small fraction of free (not virus-bound) $\operatorname{lgG}(\sim 10-20 \%)$ that will interact with mucins at any moment in time. 3,12

1,000 -fold reduced $D_{\text {eff }}$ compared with that in buffer). ${ }^{11,16}$ However, both virions readily penetrate more $\mathrm{pH}$-neutral mucus, similar to mucus secretions exposed to alkaline semen or depleted of lactobacilli, such as in women with bacterial vaginosis. ${ }^{45}$ The ability for IgG-mucin interactions to trap virions ( $\sim 1500$-fold reduced $D_{\text {eff }}$ compared with that in buffer) may therefore provide a crucial mechanism to effectively reinforce the mucus barrier. The extent of reduction in virus mobility observed here contrasts sharply with observations by Shukair et al., ${ }^{46}$ who reported an activity in genital mucus specimens from HIV-negative women (i.e., free of virusspecific IgG) that hindered HIV mobility by only a few fold on average. The mucus layer coating the female reproductive tract may be only $\sim 50-100 \mu \mathrm{m}$ thick and readily penetrated by virions within minutes even if they are slowed a few fold in mucus compared to their speed in buffer. ${ }^{11}$ Thus, to effectively reduce the flux of virions reaching target cells, it is likely essential for virions to be slowed by orders of magnitude.

Trapping virions in genital tract mucus should markedly reduce heterosexual transmission of viral infections. Women acquire many of the major sexually transmitted viral infections (e.g., HIV, HPV, and HSV) at rates on the order of 1 per 1001,000 sex acts on average (see Supplementary Text). This suggests that few, if any, of these virions are able to infect target cells per intercourse, which reflects a combination of the diffusional and other innate barrier properties of genital secretions. Therefore, any reduction in the flux of virions that reach target cells should proportionally reduce transmission rates. Indeed, the effectiveness of the IgG-mucin interactions reported here may be reflected in part by the clinical observation that HSV vaginitis is generally less common than

HSV infection of the anogenital skin (vulva and groin), which are not protected by genital mucus secretions. Blocking initial infection altogether, rather than attempting to clear initial infections, may be especially critical for infections that are difficult, if not impossible, to cure once established (e.g., HSV, HIV). Our findings not only underscore the importance of monitoring both mucosal and systemic immune responses but also motivate developing vaccines that elicit sufficient secreted $\mathrm{Ab}$ to trap pathogens in mucus in addition to eliciting neutralizing titers of systemic $\mathrm{Ab}$. In the recent gD2-AS04 HSV vaccine trial, ${ }^{47,48}$ protective efficacy was initially observed in seronegative women but not in men or seropositive women, and a larger study of seronegative women revealed only moderate efficacy against HSV-1 ( $\sim 35 \%$ efficacy against HSV1 infection and $58 \%$ efficacy against HSV-1 genital disease) but interestingly no protection against HSV-2. In both studies, the vaccine elicited neutralizing serum Ab against HSV as well as HSV-specific cellular immune responses in all women and men. However, because mucosal levels of $\mathrm{Ab}$ were not monitored, it remains unclear whether what little protection was observed could have correlated with mucosal Ab response. It is likely that generating sufficient mucosal Ab levels remains a major bottleneck to developing an effective HSV vaccine. Inducing secreted $\mathrm{Ab}$ that bind to "non-neutralizing" surface epitopes should trap pathogens as effectively as those that bind to neutralizing epitopes, a prospect that may broaden potential antigen targets for vaccine development, especially against virions with rapidly evolving neutralizing epitopes.

Fc-mediated trapping of pathogens in mucus, which directly blocks infections at the portals of entry, may represent an exceptionally potent mechanism by which the immune system can rapidly adapt and reinforce multiple mucosal surfaces against diverse and rapidly evolving pathogens. In pilot studies, we found lipopolysaccharide-specific monoclonal IgG, but not control IgG, immobilized individual Salmonella typhimurium in mucus secretions lining mouse gastrointestinal tract tissues without inhibiting the flagella beating apparatus and without causing aggregation, i.e., independent of the classical, aggregation-based mechanism of immune exclusion. The observed trapping in both CVM (predominantly Muc5b mucins) and gastrointestinal mucus (Muc2 mucins) suggests that the molecular basis for Fc-mucin affinity may be common among major secreted mucins-the long densely glycosylated fibers that form mucus gels-and possibly mediated by glycans, as sugars represent the major constituent of mucins (up to $80 \%$ by dry weight ${ }^{49}$ ). Nevertheless, significant technical challenges remain in elucidating the precise entities on mucins responsible for the observed Fc-mucin affinity, because biochemical perturbations of the mucus gel typically destroy the native mucin mesh microstructure and gel viscoelasticity ${ }^{50}$ needed to trap pathogens in the gel. Improved understanding of the molecular basis of Fc-mucin affinity will likely offer critical insight into understanding and identifying subpopulations with greater susceptibility to infection, as well as therapeutic strategies to enhance this mucosal immune-protective mechanism. 


\section{METHODS}

Culture and purification of fluorescent HSV-1. The HSV-1 mutant $166 \mathrm{v},{ }^{51}$ encoding a VP22-GFP tegument protein packaged into HSV-1 at relatively high copy numbers, ${ }^{52}$ was kindly provided by Richard Courtney and used in all microscopy and ELISA studies. The addition of GFP to the VP22 protein appears to have no deleterious effects on viral replication, ${ }^{51}$ and the fluorescence of $166 \mathrm{v}$ was consistently more intense than that of HSV-1 mutants encoding other GFP fusion proteins. $166 \mathrm{v}$ was expanded at a multiplicity of infection of three on confluent monolayers of HaCat cells maintained in Dulbecco's modified Eagle's medium (Life Technologies, Grand Island, NY) supplemented with $5 \%$ fetal bovine serum, $1 \times \mathrm{L}$-glutamine, and $1 \times$ Penicillin/Streptomycin. Culture medium was collected $16-18 \mathrm{~h}$ post infection and twice centrifuged at $1,000 \mathrm{~g}$ for $5 \mathrm{~min}$ to remove cell debris. The resulting supernatant was split into $30-\mathrm{ml}$ aliquots and precipitated overnight with a PEG/salt solution. Briefly, $10 \mathrm{ml}$ of $1.55 \mathrm{M}$ $\mathrm{NaCl}$ was added to $30 \mathrm{ml}$ of crude virus supernatant, followed by $10 \mathrm{ml}$ of $40 \%$ PEG 8000 (Sigma, St. Louis, MO). After an overnight incubation at $4{ }^{\circ} \mathrm{C}$, the virus/PEG solution was centrifuged at $2,555 \mathrm{~g}$ and $4{ }^{\circ} \mathrm{C}$ for $1 \mathrm{~h}$. The virus pellet was then resuspended in $1 \times \mathrm{PBS}$ (phosphate-buffered saline) and centrifuged through a continuous 20 $50 \%(\mathrm{w} / \mathrm{w})$ sucrose in PBS gradient for $1 \mathrm{~h}$ at $74,119 \mathrm{~g}$. The resulting virus band was further purified by diluting 1:5 in PBS, layered over $30 \%$ $(\mathrm{w} / \mathrm{w})$ sucrose in PBS, and centrifuging for $1.5 \mathrm{~h}$ at $83,472 \mathrm{~g}$ to pellet the virus for further purification. Purified virus pellet was resuspended in PBS and stored as single-use aliquots at $-80^{\circ} \mathrm{C}$.

CVM collection and characterization. CVM collection was performed as published previously. ${ }^{11,16}$ Briefly, undiluted CVM secretions, averaging $0.3 \mathrm{~g}$ per sample, were obtained from women of reproductive age, ranging from 20 to 32 years old ( $27.4 \pm 0.9$ years, mean \pm s.e.m.), by using a self-sampling menstrual collection device following protocols approved by the Institutional Review Board of the University of North Carolina-Chapel Hill. Informed consent of participants was obtained after the nature and possible consequences of the study were explained. Participants inserted the device into the vagina for at least $30 \mathrm{~s}$, removed it, and placed it into a $50-\mathrm{ml}$ centrifuge tube. Samples were centrifuged at $230 \mathrm{~g}$ for $2 \mathrm{~min}$ to collect the secretions. Aliquots of $\mathrm{CVM}$ for lactic acid and $\mathrm{Ab}$ measurements (diluted 1:5 with $1 \times \mathrm{PBS}$ and stored at $-80^{\circ} \mathrm{C}$ ) and slides for Gram staining were prepared immediately, and the remainder of the sample was stored at $4{ }^{\circ} \mathrm{C}$ until microscopy, typically within a few hours. Samples were collected at random times throughout the menstrual cycle, and cycle phase was estimated based on the last menstrual period date normalized to a 28day cycle. No samples were ovulatory based on visual observation (none exhibited spinnbarkeit). Samples that were non-uniform in color or consistency were discarded. Donors stated that they had not used vaginal products nor participated in unprotected intercourse within 3 days before donating. All samples had $\mathrm{pH}<4.5$; none had bacterial vaginosis based on Gram staining and Nugent scoring, following scoring criteria described previously. ${ }^{53}$ For lactic acid and $\mathrm{Ab}$ measurements, CVM aliquots were thawed and centrifuged for $2 \mathrm{~min}$ at $21,130 \times g$ to obtain cell-free supernatant containing lactic acid and Ab. Lactic acid content was measured using a D-/L-lactic acid kit (R-Biopharm, Darmstadt, Germany) according to the manufacturer's protocol but adapted to a 96-well format. Methods for Ab measurements are detailed in the Supplementary Materials and Methods.

Preparation and characterization of anti-HSV-1 Ab. Anti-HSV-1 IgG was affinity-purified from intravenous immunoglobulin and used to prepare anti-HSV-1 $\mathrm{F}(\mathrm{ab})_{2}$ and deglycosylated anti-HSV-1 IgG. Detailed methods are provided in the Supplementary Materials and Methods.

Neutralization assay. Purified HSV-1 ( 550 plaque-forming units; $5 \mu \mathrm{l})$ was incubated with $95 \mu \mathrm{l}$ of HSV-1-specific IgG solution at different final concentrations for $1 \mathrm{~h}$ with end-over-end mixing. The mixture was then diluted with $210 \mu$ of media, of which duplicate $150 \mu$ l aliquots were transferred to confluent Vero cell monolayers in a six-well plate. Plates were incubated at $37^{\circ} \mathrm{C}$ for $1 \mathrm{~h}$ with periodic rocking to ensure that the plates did not dry out, before the HSV-1/Ab mixture was aspirated off and wells were washed with $2 \mathrm{ml}$ of PBS. The plates were then incubated for 3 days at $37^{\circ} \mathrm{C}$ in $2 \%$ carboxymethyl cellulose in Eagle's minimal essential medium supplemented with $1 \times$ L-glutamine and $1 \times$ Penicillin/Streptomycin, before staining with $1 \%$ crystal violet solution, and the resulting plaques were manually counted and compared with control wells to determine the extent of neutralization.

FRAP. FRAP was performed following methods similar to those described by Olmsted et al. ${ }^{12}$ Fluorescein isothiocyanate-labeled Ab was added to mucus or saline at $10 \% \mathrm{v} / \mathrm{v}$ and gently stirred to achieve uniform distribution. FRAP experiments were conducted using an LSM 510 confocal microscope (Carl Zeiss Microscopy, LLC, Thornwood, NY), with a roughly $40 \times 40 \mu \mathrm{m}^{2}$ region of interest (ROI) bleached with $405 / 488$ lasers at $100 \%$ laser intensity over $\sim 3 \mathrm{~s}$. For mucus samples, ROIs were chosen to minimize epithelial cells within the bleached volume. After bleaching, the fluorescence intensity of $\mathrm{Ab}$ was monitored over time with background subtracted out and values adjusted for photobleaching of the entire image over time based on control ROIs.

Multiple particle tracking of HSV-1 in CVM. To mimic neutralization of CVM by alkaline seminal fluid, we titrated CVM to $\mathrm{pH}$ 6.8-7.1 using small volumes $(\sim 3 \% \mathrm{v} / \mathrm{v})$ of $3 \mathrm{~N} \mathrm{NaOH}$ and confirmed $\mathrm{pH}$ using a micro $\mathrm{pH}$ electrode (Microelectrodes, Bedford, $\mathrm{NH}$ ) calibrated to $\mathrm{pH}$ 4,7 , and 10 buffers. Samples were either untreated or treated by the addition of known amounts of purified anti-HSV-1 IgG or control (anti-biotin) IgG. Control beads consisted of red or green fluorescent $200 \mathrm{~nm}$ carboxyl-modified PS particles (Molecular Probes, Eugene, $\mathrm{OR}$ ), either uncoated (PS; muco-adhesive) or covalently conjugated with a low molecular weight $(2 \mathrm{kDa})$, amine-functionalized PEG (Rapp Polymere, Tuebingen, Germany) to produce coated particles (PS-PEG; muco-inert), as previously described. ${ }^{15}$ Fluorescent virions or control beads (approximately $10^{8}-10^{9}$ particles $\mathrm{ml}^{-1}$ ) were added at $5 \% \mathrm{v} / \mathrm{v}$ to $20 \mu \mathrm{l}$ of CVM placed in a custom-made glass chamber and incubated for $1 \mathrm{~h}$ at $37^{\circ} \mathrm{C}$ before microscopy. The translational motions of the particles were recorded using an EMCCD camera (Evolve 512; Photometrics, Tucson, AZ) mounted on an inverted epifluorescence microscope (AxioObserver D1; Zeiss, Thornwood, NY), equipped with an Alpha Plan-Apo $100 \times / 1.46$ NA objective, environmental (temperature and $\mathrm{CO}_{2}$ ) control chamber, and an LED light source (Lumencor Light Engine DAPI/GFP/543/623/690). Videos $(512 \times 512$, 16-bit image depth) were captured with MetaMorph imaging software (Molecular Devices, Sunnyvale, CA) at a temporal resolution of $66.7 \mathrm{~ms}$ and spatial resolution of $10 \mathrm{~nm}$ (nominal pixel resolution $0.156 \mu \mathrm{m}$ per pixel) for $20 \mathrm{~s}$. The tracking resolution was determined by tracking the displacements of particles immobilized with a strong adhesive, following a previously described method. ${ }^{54}$ Particle trajectories were analyzed using the MetaMorph software as described previously; ${ }^{11,15,16}$ image contrast was adjusted to improve particle visibility, but the same contrast level was applied throughout the entire video and did not bias toward any particle population. Subpixel tracking resolution is obtained by determining the precise location of the particle centroid by light-intensity-weighted averaging of neighboring pixels. Trapped particles were defined as those with effective diffusivity $\left(D_{\text {eff }}\right)<0.01 \mu \mathrm{m}^{2} \mathrm{~s}^{-1}$ at a timescale $(\tau)$ of $1 \mathrm{~s}$ (i.e., particles move less than their diameter within $1 \mathrm{~s}$ ). In a subset of experiments, we confirmed that particles defined as trapped over the course of $20 \mathrm{~s}$ based on this criterion remain confined to the same locations over $>15 \mathrm{~min}$. The slope $\alpha$ of the log-log mean square displacement $(<\mathrm{MSD}>$ ) vs. timescale plot provides a further measure of particle mobility: $\alpha=1$ for pure unobstructed Brownian diffusion, e.g., for particles in water, $\alpha$ becomes smaller as obstruction to particle diffusion increases, and $\alpha$ is zero for permanently trapped particles. At 
least five independent experiments in CVM from different donors, with $n \geqslant 100$ particles per experiment, were performed for each condition. For a subset of donors, similar observations were made at least twice in samples obtained on separate days to ensure reproducibility, but only one sample was used for analysis.

Mouse vaginal HSV-2 challenge model. All experiments conducted with mice were performed in accordance with the protocols approved by the Johns Hopkins University Animal Care and Use Committee satisfying the requirements of the E.E.C. Guidelines (1986) and US Federal Guidelines (1985). Female CF-1 mice (6-8 weeks old; Harlan, Frederick, MD) were treated with Depo-Provera (medroxyprogesterone acetate, $2.5 \mathrm{mg}$ per mouse) by subcutaneous injection into the right flank 6-8 days before use. Depo-Provera synchronizes mice in a prolonged diestrus-like state, in which the vaginal epithelium thins and susceptibility of the tissue to infection increases. ${ }^{55}$ DepoProvera-treated mice were randomly divided into groups of 10 . The mouse vagina is $\mathrm{pH}$ neutral; ${ }^{22}$ therefore, we did not attempt to modify vaginal $\mathrm{pH}$ before inoculation. Inocula were prepared by mixing HSV-2 (final dose $2 \mathrm{ID}_{50}$; strain G, ATCC, Manassas, VA) with medium or different concentrations of control (anti-biotin) or anti-gG mouse monoclonal IgG (8.F.141; Santa Cruz Biotechnology, Santa Cruz, CA) and incubating for $1 \mathrm{~h}$ at $37^{\circ} \mathrm{C}$. Mice were inoculated with $20 \mu \mathrm{l}$ of HSV-2 solution, delivered to the vagina using a $50-\mu \mathrm{l}$ Wiretrol (Drummond, Broomall, PA), and fire-polished to avoid damage to the vaginal epithelium. In some studies, the mouse vagina was gently washed with $\sim 10 \mathrm{ml}$ of normal saline delivered at $1 \mathrm{ml} \mathrm{min}^{-1}$ through a smooth ball-tipped gavage needle connected to a syringe pump, before HSV-2 challenge. Removal of mucus by this process was measured using a fluorimetric mucin assay performed, as previously described, ${ }^{56}$ on fluid recovered from a $50 \mu$ l vaginal lavage with normal saline of mice with and without the gentle vaginal washing. Importantly, the gentle wash did not damage the vaginal epithelium, as confirmed by microscopy with a fluorescence-based dead cell stain (YOYO-1) that assesses membrane integrity, ${ }^{55}$ compared with conventional lavage and/or vaginal swabbing with a cotton tip, which induced significant epithelial damage. YOYO-1 has been previously used to reveal toxicity caused by detergent-based microbicides that led to increased susceptibility to HSV infection. ${ }^{55}$ Tissue sectioning and hematoxylin and eosin staining were performed by the Animal Histopathology lab at the University of North Carolina-Chapel Hill. Infection was assayed 3 days post inoculation by detection of virus in vaginal lavages. Briefly, $50 \mu \mathrm{l}$ of the medium was pipetted in and out of the vagina 20 times, diluted to $0.2 \mathrm{ml}$, and placed on target cells (ELVIS HSV Test System; Diagnostic Hybrids, Athens, $\mathrm{OH}$ ); infected cells (foci) were identified 1 day later, following the manufacturer's protocol. Scores for virus shedding were assigned on a scale of $0-4$ based on the approximate density of foci observed (" 0 ": 0 ; “0.5": <100; "1": 100-500; "2": 5001,000 ; " 3 ": $1,000+;$ " 4 ": saturated). At least three independent experiments were performed for each condition, with $n=10$ animals per experimental group ( $n \geqslant 30$ total).

Statistical analysis. Correlation between endogenous anti-HSV-1 IgG levels and average particle or virus $D_{\text {eff }}$ in individual CVM samples was measured using Pearson's correlation coefficient $(r)$. Statistical comparisons were limited to two groups (test group compared with the appropriate control group performed during the same experiment). Fisher's exact test was used to determine the statistical significance of reductions in the percentage of mice infected. A two-tailed Student's $t$-test (paired for comparisons of Abtreated vs. native CVM for the same CVM samples) was used for all other comparisons. Differences were deemed significant at an $\alpha$ level of 0.05 . All values are reported as mean \pm s.e.m. unless otherwise indicated.

SUPPLEMENTARY MATERIAL is linked to the online version of the paper at http://www.nature.com/mi

\section{ACKNOWLEDGMENTS}

We thank the Clinical Microbiology and Immunology Lab in the Department of Pathology at the University of North Carolina-Chapel Hill for assistance with Nugent scoring. This work was supported by the National Institutes of Health grants R21Al093242 (to S.K.L.), U19AI096398 (to S.K.L. and R.C.) and 1F32Al102535 (to Y.-Y.W.), National Science Foundation CAREER Award DMR-1151477 (to S.K.L.), Gates Grand Challenges Round 5 OPP1024615 (to S.K.L.), and startup funds from the Eshelman School of Pharmacy and Lineburger Cancer Center at University of North Carolina-Chapel Hill. The content is solely the responsibility of the authors and does not necessarily represent the official views of the National Institutes of Health.

\section{DISCLOSURE}

The authors declared no conflict of interest.

c 2014 Society for Mucosal Immunology

\section{REFERENCES}

1. Li, Z., Palaniyandi, S., Zeng, R., Tuo, W., Roopenian, D.C. \& Zhu, X. Transfer of $\operatorname{lgG}$ in the female genital tract by MHC class I-related neonatal Fo receptor ( $\mathrm{FCRn}$ ) confers protective immunity to vaginal infection. Proc. Natl. Acad. Sci. USA 108, 4388-4393 (2011).

2. Usala, S.J., Usala, F.O., Haciski, R., Holt, J.A. \& Schumacher, G.F. IgG and IgA content of vaginal fluid during the menstrual cycle. J. Reprod. Med. 34, 292-294 (1989).

3. Cone, R.A. Mucus. In Handbook of Mucosal Immunology, 3rd edn. Ogra, P.L. \& Mestecky, J. \& Lamm, M.E. \& Strober, W. \& Bienenstock, J. \& McGhee, J.R., eds) 43-64 (Academic Press, San Diego, CA, USA, 1999).

4. Hill, J.A. \& Anderson, D.J. Human vaginal leukocytes and the effects of vaginal fluid on lymphocyte and macrophage defense functions. Am. J. Obstet. Gynecol. 166, 720-726 (1992).

5. Schumacher, G.F. Immunology of spermatozoa and cervical mucus. Hum. Reprod. 3, 289-300 (1988).

6. Kresge, K.J. The Mysteries of Protection. IAVI Report, Vol. 13, Number 5 [Internet] (2009).

7. Rerks-Ngarm, S. et al. Vaccination with ALVAC and AIDSVAX to prevent HIV-1 infection in Thailand. N. Engl. J. Med. 361, 2209-2220 (2009).

8. Brockman, M.A. \& Knipe, D.M. Herpes simplex virus as a tool to define the role of complement in the immune response to peripheral infection. Vaccine 26 (Suppl 8), 194-199 (2008).

9. Hook, L.M., Lubinski, J.M., Jiang, M., Pangburn, M.K. \& Friedman, H.M. Herpes simplex virus type 1 and 2 glycoprotein $C$ prevents complementmediated neutralization induced by natural immunoglobulin $\mathrm{M}$ antibody. J. Virol. 80, 4038-4046 (2006).

10. Lubinski, J., Wang, L., Mastellos, D., Sahu, A., Lambris, J.D. \& Friedman, H.M. In vivo role of complement-interacting domains of herpes simplex virus type 1 glycoprotein gC. J. Exp. Med. 190, 1637-1646 (1999).

11. Lai, S.K. et al. Human immunodeficiency virus type 1 is trapped by acidic but not by neutralized human cervicovaginal mucus. J. Virol. 83, 11196-11200 (2009)

12. Olmsted, S.S., Padgett, J.L., Yudin, A.I., Whaley, K.J., Moench, T.R. \& Cone, R.A. Diffusion of macromolecules and virus-like particles in human cervical mucus. Biophys. J. 81, 1930-1937 (2001).

13. Cole, A.M. Innate host defense of human vaginal and cervical mucosae. Curr. Top. Microbiol. Immunol. 306, 199-230 (2006).

14. Doss, M., White, M.R., Tecle, T. \& Hartshorn, K.L. Human defensins and LL-37 in mucosal immunity. J. Leukoc. Biol. 87, 79-92 (2010).

15. Lai, S.K. et al. Rapid transport of large polymeric nanoparticles in fresh undiluted human mucus. Proc. Natl. Acad. Sci. USA 104, 1482-1487 (2007).

16. Lai, S.K., Wang, Y.Y., Hida, K., Cone, R. \& Hanes, J. Nanoparticles reveal that human cervicovaginal mucus is riddled with pores larger than viruses. Proc. Natl. Acad. Sci. USA 107, 598-603 (2010). 
17. Hamburger, A.E., Bjorkman, P.J. \& Herr, A.B. Structural insights into antibody-mediated mucosal immunity. Curr. Top. Microbiol. Immunol. 308, 173-204 (2006).

18. Mantis, N.J., Rol, N. \& Corthesy, B. Secretory IgA's complex roles in immunity and mucosal homeostasis in the gut. Mucosal Immunol. 4 , 603-611 (2011).

19. Berzofsky, J.A., Berkower, I.J. \& Epstein, S.L. Antigen-antibody interactions and monoclonal antibodies. In Fundamental Immunology (Paul, W.E., ed) 421-465 (The Raven Press, New York, NY, USA, 1993).

20. Saltzman, W.M., Radomsky, M.L., Whaley, K.J. \& Cone, R.A. Antibody diffusion in human cervical mucus. Biophys. J. 66, 508-515 (1994).

21. Ha, S. et al. Isolation and characterization of lgG1 with asymmetrical FC glycosylation. Glycobiology 21, 1087-1096 (2011).

22. Meysick, K.C. \& Garber, G.E. Interactions between Trichomonas vaginalis and vaginal flora in a mouse model. J. Parasitol. 78, 157-160 (1992).

23. Ey, P.L., Russell-Jones, G.J. \& Jenkin, C.R. Isotypes of mouse IgG-I. Evidence for 'non-complement-fixing' IgG1 antibodies and characterization of their capacity to interfere with IgG2 sensitization of target red blood cells for lysis by complement. Mol. Immunol. 17, 699-710 (1980).

24. Michaelsen, T.E., Kolberg, J., Aase, A., Herstad, T.K. \& Hoiby, E.A. The four mouse IgG isotypes differ extensively in bactericidal and opsonophagocytic activity when reacting with the P1.16 epitope on the outer membrane PorA protein of Neisseria meningitidis. Scand. J. Immunol. 59, 34-39 (2004).

25. Neuberger, M.S. \& Rajewsky, K. Activation of mouse complement by monoclonal mouse antibodies. Eur. J. Immunol. 11, 1012-1016 (1981).

26. Akiyama, Y., Lubeck, M.D., Steplewski, Z. \& Koprowski, H. Induction of mouse IgG2a- and IgG3-dependent cellular cytotoxicity in human monocytic cells (U937) by immune interferon. Cancer Res. 44, 5127-5131 (1984).

27. Kipps, T.J., Parham, P., Punt, J. \& Herzenberg, L.A. Importance of immunoglobulin isotype in human antibody-dependent, cell-mediated cytotoxicity directed by murine monoclonal antibodies. J. Exp. Med. 161, 1-17 (1985)

28. Zeitlin, L., Whaley, K.J., Hegarty, T.A., Moench, T.R. \& Cone, R.A. Tests of vaginal microbicides in the mouse genital herpes model. Contraception $\mathbf{5 6}$, 329-335 (1997).

29. Stefanidou, M. et al. Herpes simplex virus 2 (HSV-2) prevents dendritic cell maturation, induces apoptosis, and triggers release of proinflammatory cytokines: potential links to HSV-HIV synergy. J. Virol. 87, 1443-1453 (2013).

30. Yuan, W., Dasgupta, A. \& Cresswell, P. Herpes simplex virus evades natural killer T cell recognition by suppressing CD1d recycling. Nat. Immunol. 7, 835-842 (2006).

31. Jager, S., Kremer, J., Kuiken, J. \& Mulder, I. The significance of the Fc part of antispermatozoal antibodies for the shaking phenomenon in the spermcervical mucus contact test. Fertil. Steril. 36, 792-797 (1981).

32. Kremer, J. \& Jager, S. The sperm-cervical mucus contact test: a preliminary report. Fertil. Steril. 27, 335-340 (1976).

33. Phalipon, A., Cardona, A., Kraehenbuhl, J.P., Edelman, L., Sansonetti, P.J. \& Corthesy, B. Secretory component: a new role in secretory IgA-mediated immune exclusion in vivo. Immunity 17, 107-115 (2002).

34. Fahrbach, K.M., Malykhina, O., Stieh, D.J. \& Hope, T.J. Differential binding of $\operatorname{lgG}$ and IgA to mucus of the female reproductive tract. PLoS One 8 , e76176 (2013).

35. Kobayashi, K. et al. Distribution and partial characterisation of IgG FC binding protein in various mucin producing cells and body fluids. Gut 51, 169-176 (2002).

36. Johansson, M.E., Larsson, J.M. \& Hansson, G.C. The two mucus layers of colon are organized by the MUC2 mucin, whereas the outer layer is a legislator of host-microbial interactions. Proc. Natl. Acad. Sci. USA 108 (Suppl 1), 4659-4665 (2011).

37. Johansson, M.E., Thomsson, K.A. \& Hansson, G.C. Proteomic analyses of the two mucus layers of the colon barrier reveal that their main component, the Muc2 mucin, is strongly bound to the Fcgbp protein. J. Proteome Res. 8, 3549-3557 (2009).

38. Clamp, J.R. The relationship between secretory immunoglobulin A and mucus [proceedings]. Biochem. Soc. Trans. 5, 1579-1581 (1977).

39. Crowther, R., Lichtman, S., Forstner, J. \& Forstner, G. Failure to show secretory IgA binding by rat intestinal mucin. Fed. Proc. 44, 691 (1985).

40. Kobayashi, K., Blaser, M.J. \& Brown, W.R. Identification of a unique IgG Fc binding site in human intestinal epithelium. J. Immunol. 143, 2567-2574 (1989).

41. Andersch-Bjorkman, Y., Thomsson, K.A., Holmen Larsson, J.M., Ekerhovd, E. \& Hansson, G.C. Large scale identification of proteins, mucins, and their O-glycosylation in the endocervical mucus during the menstrual cycle. Mol. Cell. Proteomics 6, 708-716 (2007).

42. Dasari, S. et al. Comprehensive proteomic analysis of human cervicalvaginal fluid. J. Proteome Res. 6, 1258-1268 (2007).

43. Shaw, J.L., Smith, C.R. \& Diamandis, E.P. Proteomic analysis of human cervico-vaginal fluid. J. Proteome Res. 6, 2859-2865 (2007).

44. Tang, L.J. et al. Proteomic analysis of human cervical-vaginal fluids. J. Proteome Res. 6, 2874-2883 (2007).

45. Amsel, R., Totten, P.A., Spiegel, C.A., Chen, K.C., Eschenbach, D. \& Holmes, K.K. Nonspecific vaginitis. Diagnostic criteria and microbial and epidemiologic associations. Am. J. Med. 74, 14-22 (1983).

46. Shukair, S.A. et al. Human cervicovaginal mucus contains an activity that hinders HIV-1 movement. Mucosal Immunol. 6, 427-434 (2013).

47. Belshe, R.B. et al. Efficacy results of a trial of a herpes simplex vaccine. N. Engl. J. Med. 366, 34-43 (2012).

48. Leroux-Roels, G., Clement, F., Vandepapeliere, P., Fourneau, M., Heineman, T.C. \& Dubin, G. Immunogenicity and safety of different formulations of an adjuvanted glycoprotein $D$ genital herpes vaccine in healthy adults: a double-blind randomized trial. Hum. Vaccin Immunother. 9, 1254-1262 (2013).

49. Lai, S.K., Wang, Y.Y. \& Hanes, J. Mucus-penetrating nanoparticles for drug and gene delivery to mucosal tissues. Adv. Drug Deliv. Rev. 61, 158-171 (2009).

50. Kocevar-Nared, J., Kristl, J. \& Smid-Korbar, J. Comparative rheological investigation of crude gastric mucin and natural gastric mucus. Biomaterials 18, 677-681 (1997).

51. Elliott, G. \& O'Hare, P. Live-cell analysis of a green fluorescent protein-tagged herpes simplex virus infection. J. Virol. 73, 4110-4119 (1999).

52. Heine, J.W., Honess, R.W., Cassai, E. \& Roizman, B. Proteins specified by herpes simplex virus. XII. The virion polypeptides of type 1 strains. J. Virol. 14, 640-651 (1974).

53. Nugent, R.P., Krohn, M.A. \& Hillier, S.L. Reliability of diagnosing bacterial vaginosis is improved by a standardized method of gram stain interpretation. J. Clin. Microbiol. 29, 297-301 (1991).

54. Apgar, J., Tseng, Y., Fedorov, E., Herwig, M.B., Almo, S.C. \& Wirtz, D. Multiple-particle tracking measurements of heterogeneities in solutions of actin filaments and actin bundles. Biophys. J. 79, 1095-1106 (2000).

55. Cone, R.A., Hoen, T., Wong, X., Abusuwwa, R., Anderson, D.J. \& Moench, T.R. Vaginal microbicides: detecting toxicities in vivo that paradoxically increase pathogen transmission. BMC Infect. Dis. 6, 90 (2006).

56. Crowther, R.S. \& Wetmore, R.F. Fluorometric assay of O-linked glycoproteins by reaction with 2-cyanoacetamide. Anal. Biochem. 163, 170-174 (1987). 